\title{
ANÁLISE DOS EFEITOS DO TRATAMENTO TERMOQUÍMICO DE BORETAÇÃO, APLICADO A AÇOS COMERCIAIS DE LARGA UTILIZAÇÃO INDUSTRIAL: REVISÃO
}

\author{
Maicon Perini ${ }^{1}$, Daniela Lupinacci Villanova ${ }^{1}$ \\ 1 Programa de Pós-Graduação em Tecnologia e Engenharia dos Materiais - PPGTEM, Instituto Federal do Rio Grande do Sul, \\ Multicampi, Brasil.
}

*E-mail: eng.maiconperini@gmail.com

Recebido em: $31 / 05 / 2019$

Aceito em: 12/08/2019

\section{RESUMO}

A intensificação dos estudos, visando o entendimento pleno das variáveis que envolvem o tratamento termoquímico de boretação é de fundamental importância para que se possa obter pleno conhecimento, dessa forma será possível implantar tal técnica em níveis de escala industrial. Apesar de se tratar de uma rica área dentro do contexto da produção científica, ainda existem muitas lacunas a serem desvendadas no que diz respeito à boretação, especialmente quanto aos agentes boretantes que podem ser utilizados no tratamento, além de temperatura e tempo adequado. Tem-se por objetivo elencar o maior número possível de dados disponíveis acerca do tema proposto, a partir de uma revisão bibliográfica atualizada, o quê evidenciará que a boretação, de fato, apresenta-se como uma solução eficaz para problemas de desgastes em componentes industriais, uma vez que pode elevar em até cinco vezes a dureza superficial do aço base em seu estado normalizado, o qual é utilizado para a fabricação dos mesmos.

Palavras-chave: Boretação. Revisão bibliográfica. Aço. Dureza.

\section{Introdução}

Os requisitos para melhorar propriedades como a resistência ao desgaste, em componentes de engenharia, levaram a extenuantes estudos sobre as técnicas mais convenientes e menos dispendiosas para o endurecimento superficial de aços. As técnicas de endurecimento de superfície incluem métodos térmicos, tais como, endurecimento por chama e endurecimento por indução e métodos termoquímicos tais como cementação, nitretação e boretação [1].

Uma rápida expansão dos estudos, no campo de tratamento superficial, tem sido promovida, por conta da crescente demanda de materiais com resistência satisfatória ao desgaste [2].

$\mathrm{O}$ desgaste de componentes, utilizados em equipamentos/máquinas de uso industrial está ao lado da fadiga e corrosão, entre os problemas que com maior frequência, trazem prejuízos aos parques industriais. $\mathrm{O}$ processo de desgaste, em geral, não se mostra catastrófico, entretanto diminui a eficiência das operações em virtude de perdas crescentes de potência, aumento significativo do consumo de energia e de óleos lubrificantes, isso sem contar no tempo improdutivo que será necessário para a realização de operações de substituição de tais componentes. O segmento da manutenção industrial busca, com constância, soluções que minimizem o desgaste possibilitando um aumento do tempo entre as paradas de manutenção e reduzindo as substituições. A existência de possibilidade para a recuperação de componentes ou preparação para que suportem melhor as condições de trabalho, movem com sinergia acelerada, o estudo de novas técnicas que permitam aumentar a vida útil e reduzir custos operacionais. O desgaste representa, em vista do exposto, exacerbado significado econômico para os parques industriais [3].

Estudos recentes indicam que o boro pode ser utilizado para obter dureza e melhorar a resistência ao desgaste em superfícies de aço [4].

A boretação trata-se de um tratamento de natureza termoquímica e abrangência superficial, através do qual se busca criar uma camada de dureza elevada, sendo que esta é formada por boretos de elementos do substrato. No percurso deste tratamento, os átomos de boro difundir-se-ão para o substrato, gerando reação com o metal base, assim forma-se a desejada camada de boreto. Este tratamento termoquímico pode ser utilizado em uma extensa gama de materiais, ferrosos e não ferrosos, tornando-se uma importante alternativa à formação de uma superfície de elevada dureza, conferindo aos mais diversos componentes, alta resistência ao desgaste e reduzindo o coeficiente de atrito [3].

Tratamentos de difusão térmica de compostos de boro, usados para formar boretos de ferro, normalmente requerem temperaturas de processo de $700^{\circ} \mathrm{C} \mathrm{a} 1000^{\circ} \mathrm{C}$. O processo pode ser realizado em meio sólido, líquido ou gasoso. $\mathrm{O}$ método mais utilizado é o banho boronizante [5-6]. 
O banho boronizante contém uma fonte de boro, geralmente carboneto de boro $\left(\mathrm{B}_{4} \mathrm{C}\right)$ ou boro amorfo e um ativador para depositar boro atômico na peça de trabalho. No método do banho boronizante, o componente é depositado em um recipiente, no qual já se encontram em fase líquida a fonte de boro, bem como o ativador, na sequência o recipiente é reconduzido ao forno onde é aquecido até a temperatura exigida e pelo tempo necessário, posteriormente, o componente é retirado do banho e resfriado ao ar. Geralmente, a formação de uma monofase $\left(\mathrm{Fe}_{2} \mathrm{~B}\right)$, com morfologia dente de serra, é mais desejável do que uma camada de dupla fase com $\mathrm{FeB}$ e $\mathrm{Fe}_{2} \mathrm{~B}$, para aplicações industriais. Uma única camada de $\mathrm{Fe}_{2} \mathrm{~B}$ produz uma resistência ao desgaste superior e propriedades mecânicas mais interessantes [7].

Em particular, a boretação é o método de tratamento de superfície que gera uma camada de boreto, com dureza na faixa de $1600 \mathrm{HV}$ a $2000 \mathrm{HV}$, valendo-se da penetração e difusão do boro através da superfície. A camada de boreto também tem uma excelente resistência ao calor e à corrosão [8].

Muitas técnicas novas de boretação foram investigadas ao longo dos últimos anos, a fim de melhorar a eficiência e a qualidade do referido tratamento. Verificou-se que a cinética de difusão de boro foi aumentada quando se empregou deformação plástica na superfície do metal base, antes da boretação, ao se utilizar a técnica do banho boronizante para a realização do tratamento. Acredita-se que o aumento da espessura da camada de boreto seja resultado da deformação plástica superficial, a qual gera grãos finos, uma estrutura nanocristalina com alta energia livre e deslocamentos de alta densidade na amostra tratada [9-10].

No presente estudo, desenvolver-se-á uma metodologia para futura avaliação de propriedades mecânicas do aço, quando submetido ao tratamento termoquímico de boretação. A morfologia da camada de boreto foi caracterizada a partir da dureza, profundidade e microestrutura.

\section{Metodologia}

\subsection{Materiais de Substrato}

Poucos trabalhos científicos tratam acerca do tratamento termoquímico de boretação para o aço comercial de baixo carbono AISI1020, já para Aços com maior teor de carbono, existem boas referências teóricas para nortear o presente estudo, há uma significativa quantidade de trabalhos que tratam de boretação para o aço AISI1040, além de aços especiais como AISI P20, largamente utilizado pela indústria para fabricação de moldes para injeção de polímeros. O principal objetivo do presente estudo é compilar o maior número de dados relevantes possíveis, além de desenvolver uma metodologia adequada para avaliar os efeitos provocados às propriedades dos aços comerciais AISI1020 e AISI1045, quando estes são submetidos ao Tratamento Termoquímico de Boretação.

\subsection{Definição dos Corpos de Prova}

A fim de definir quais serão as geometrias adequadas para confecção dos corpos de prova, os quais serão utilizados para a realização do tratamento termoquímico de boretação, buscaramse, dentro do conhecimento científico produzido nesta área, informações que poderão ser úteis às definições que deverão ser tomadas quando da necessidade de realização dos experimentos que estão sendo propostos a realizar.

Analisando um trabalho publicado no ano de 2017, o qual foi desenvolvido em uma universidade chinesa, verifica-se a utilização de corpos de provas cilíndricos com $\varnothing 15 \mathrm{~mm}$ e espessura de $5 \mathrm{~mm}$, tendo as faces destes, sido lixadas até lixa 1000 mesh [11]. Já em um segundo trabalho, também publicado no ano de 2017, o qual foi desenvolvido em uma universidade brasileira, utilizaram-se corpos de prova, com seção quadrada, medindo $20 \mathrm{~mm} \times 20 \mathrm{~mm}$ e espessura de $5 \mathrm{~mm}$, tendo as faces destes, sido lixadas até lixa 600 mesh [3]. Por fim, em trabalho que data do ano de 2004, desenvolvido em universidade turca, optou-se pela utilização de corpos de prova, com seção quadrada, medindo $10 \mathrm{mmx} 10 \mathrm{~mm}$ e espessura de $8 \mathrm{~mm}$, tendo as faces destes, sido lixadas até lixa 1000 mesh [16].

\subsection{Definições das Técnicas do Tratamento de Boretação}

O tratamento termoquímico de boretação, efetuado em universidade mexicana no ano de 2016, foi realizado a partir da colocação dos corpos de prova do material selecionado, em uma caixa cilíndrica, selada, onde os corpos de prova fiquem imersos em um pó, que apresente características próprias para produzir a difusão do boro para os corpos de prova, no caso do trabalho citado, utilizou-se pó com a seguinte composição: $20 \% \mathrm{~B}_{4} \mathrm{C}$ (Doador); $10 \% \mathrm{KBF}_{4}$ (Ativador) e $30 \% \mathrm{SiC}$ (Diluente). O tratamento, em forno, deve ocorrer à temperatura de $900^{\circ} \mathrm{C}, 950^{\circ} \mathrm{C}$ ou $1000^{\circ} \mathrm{C}$, durante um período de $4 \mathrm{~h}, 6 \mathrm{~h}$ ou $8 \mathrm{~h}$. Neste estudo o material analisado era o aço AISI1045 [12].

Um segundo trabalho analisado, realizado na Turquia no ano de 2005, utilizou-se da mesma técnica descrita anteriormente, apenas cita-se que a caixa cilíndrica utilizada fora fabricada em aço inoxidável, o pó boretante utilizou-se da mesma composição citada anteriormente e o tratamento, em forno, ocorreu às temperaturas de $800^{\circ} \mathrm{C}, 875^{\circ} \mathrm{C}$ e $900^{\circ} \mathrm{C}$, durante um período de $2 \mathrm{~h}, 4 \mathrm{~h}, 6 \mathrm{~h}$ ou $8 \mathrm{~h}$. Neste estudo analisou-se o comportamento de dois materiais: AISI 1040 e AISI P20 [13].

Por fim, em outro trabalho turco do ano de 1999, houve a utilização de um pó comercial, com o intuito de realizar o tratamento de boretação, trata-se de um composto de origem germânica, conhecido como EKabor ${ }^{\circledR}$, uma única temperatura de tratamento, em forno, foi utilizada, $900^{\circ} \mathrm{C}$, durante os seguintes períodos: 2h, 3h, $4 \mathrm{~h}$ e 5h. Neste estudo analisou-se o comportamento de vários materiais, tais como: AISI 1020, AISI 1030, AISI 1040 e AISI 1050 [14]. 


\subsection{Caracterização da Camada de Boreto}

A camada de boreto formada, após o tratamento termoquímico, pode ser caracterizada através da técnica de difração de raio-X, empregando-se um difratômetro XRD-6000. Também se avaliou a microdureza e a tenacidade da camada de boreto formada, para isso utilizou-se um indentador Vicker e, por fim, com o intuito de avaliar a profundidade da camada de boreto formada, utilizou-se um instrumento de medição digital acoplado a um microscópio ótico Olympus BHM-313, a qual forneceu adequada resolução para a medição necessária [13].

Em um segundo estudo, a caracterização da camada de boreto também se deu através da técnica da difração de raio-x, entretanto, neste trabalho, há um detalhamento maior dos pormenores particularizados da técnica, onde utiliza-se uma radiação Co K $\alpha$, com um comprimento de onda de $1.7902 \AA$ em um intervalo angular de $2 \theta$ e variação $20^{\circ}-80^{\circ}$. Neste trabalho, a profundidade da camada produzida foi medida com um micrômetro ótico acoplado a um microscópio ótico Olympus B071, a questão da microdureza e tenacidade da camada de boreto formada também foi avaliada com um indentador Vicker, aplicando-se uma carga de 3N [15].

Por fim, em um terceiro trabalho avaliado, a análise de difração de raio-x foi realizada utilizando um difratômetro Rigaku Gergeflex, radiação $\mathrm{Cu} K \alpha$, cujo comprimento de onda estabelecido era $1.54 \AA$ em um intervalo angular de $2 \theta$ e variação $20^{\circ}-100^{\circ}$, esta análise serviu para prover a caracterização da camada de boreto formada. Neste estudo, a espessura da camada de boreto foi medida usando um micrômetro acoplado a um microscópio ótico e os valores foram determinados a partir da média de seis medidas, já quanto à questão da determinação da microdureza, utilizou-se a média de seis medidas transversais à superfície da camada de boretos, em uma profundidade de $30 \mu \mathrm{m}$, usando uma carga de 50gf, tendo como suporte para determinação da microdureza Vickers um microdurômetro digital Buehler modelo 1600-6300 [3].

\section{Resultados e discussões}

\subsection{Caracterização da Microestrutura}

Em um importante trabalho que se avaliou, com o intuito de escrever o presente artigo de revisão, após a realização do tratamento de boretação, identificou-se a formação de uma camada dupla composta por $\mathrm{FeB}+\mathrm{Fe}_{2} \mathrm{~B}$ na superfície do aço tratado, isso foi comprovado através da técnica da difração de raio-X, apresentada na Figura 01. No caso específico que está sendo apresentado, a camada de FeB encontra-se na superfície e a camada de $\mathrm{Fe}_{2} \mathrm{~B}$ está localizada entre o substrato do aço base e a camada superficial mencionada, formada por FeB [13]. Neste mesmo trabalho, pode-se verificar a morfologia da camada de boretos formada, a qual se assemelha a dentes serrados, permitindo excelente adesão entre a camada formada e o substrato, a Figura 02 apresenta em detalhes este resultado. Por fim, este importante trabalho científico, ainda explana acerca da profundidade das camadas de boretos formadas, além da dureza superficial obtida nos corpos de prova após a realização do tratamento termoquímico de boretação. Em maiores detalhes essas informações estão sintetizadas na Figura 03 e Figura 04, respectivamente [13].

\subsection{Discussões Acerca do Tema}

Ao longo da análise bibliográfica que foi realizada, com o intuito de aprofundar e proporcionar bases científicas para a realização de um trabalho acerca do tratamento termoquímico de boretação foi possível verificar que, importantes resultados estão sendo obtidos, em diferentes partes do mundo e por diferentes pesquisadores, no que tange a melhora de propriedades superficiais de materiais, especialmente o aço. A Tabela 01 faz uma compilação dos principais artigos científicos apresentados sobre o tema e os respectivos resultados obtidos, quando da realização do tratamento termoquímico de boretação, sob condições que também são detalhadas. Tratam-se de resultados que evidenciam um aumento de dureza superficial em até 5 vezes o valor originalmente encontrado nos materiais de substrato, o quê certamente se traduz em grandes ganhos na constante batalha travada contra o desgaste de componentes mecânicos.

Os resultados apresentados na Figura 02 retratam a condição almejada para a camada de boretos formada. A forma de dentes serrados proporciona, entre a camada e o material de substrato, uma excelente adesividade, permitindo que tensões de cisalhamento sejam aplicadas sem causar danos.

Já os gráficos apresentados na figura 03, mostram informações, de extrema importância, para a definição de parâmetros a serem utilizados quando da realização do tratamento termoquímico de boretação. Torna-se possível, através de sua leitura, identificar a espessura da camada de boretos que se formará dependendo do tempo e da temperatura utilizados.

Por fim, deparamo-nos com uma compilação de informações, apresentadas no gráfico da figura 04, no qual a dureza (HV) é evidenciada ao longo da profundidade da camada de boretos. Ressalta-se a magnitude de dureza para o aço AISI P20, cuja a dureza nominal é de 300HV mas, após a boretação, atinge-se valores na ordem de $1650 \mathrm{HV}$, na superfície. 

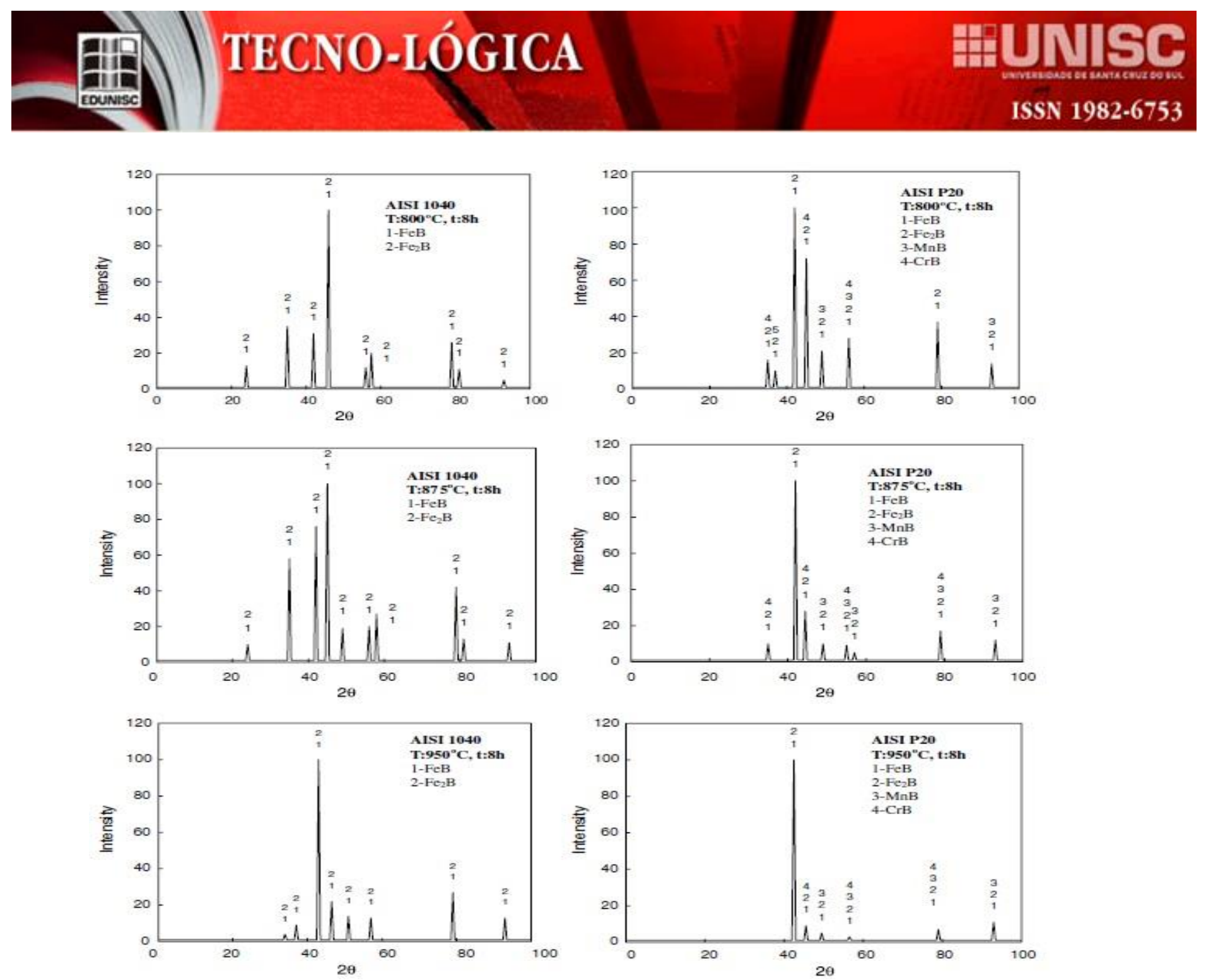

Figura 01 - Padrões de difração de raio-X, identificando a presença de boretos de ferro no aço AISI 1040 e AISI P20 [13].

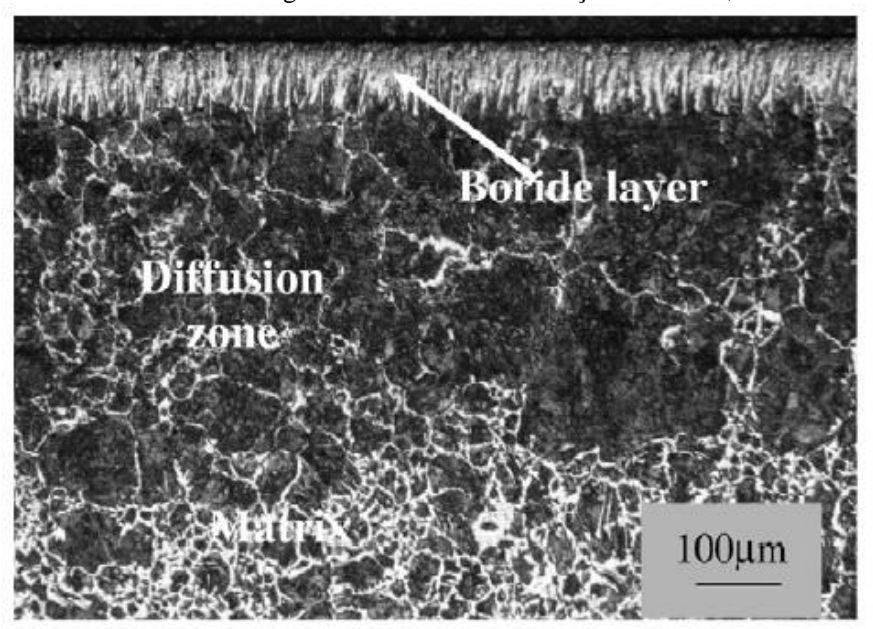

(a)

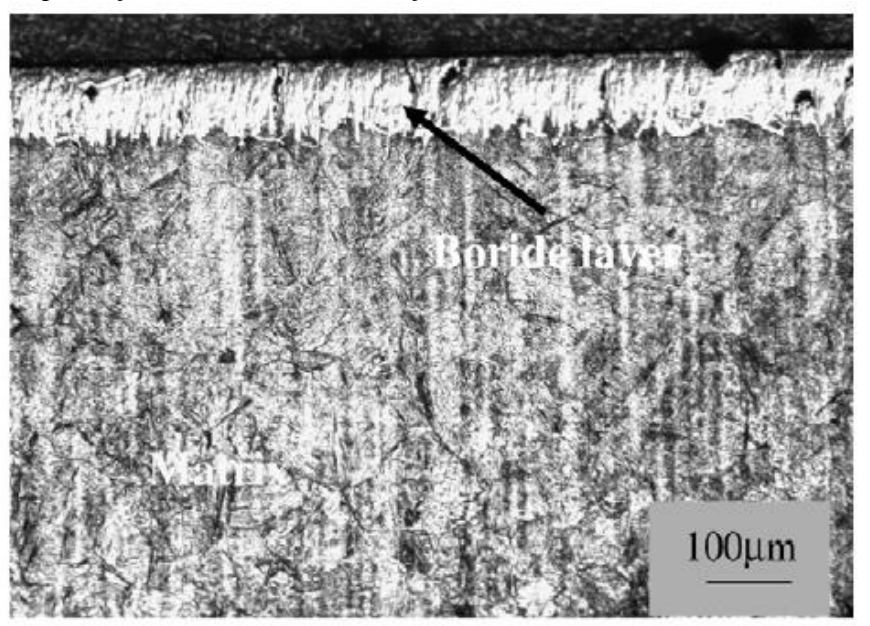

(b)

Figura 02 - Vista de corte transversal do aço boretado AISI 1040 (a); Vista de corte transversal do aço boretado AISI P20 (b). Temperatura de forno $900^{\circ} \mathrm{C}$ em um período de 6 horas [13]. 


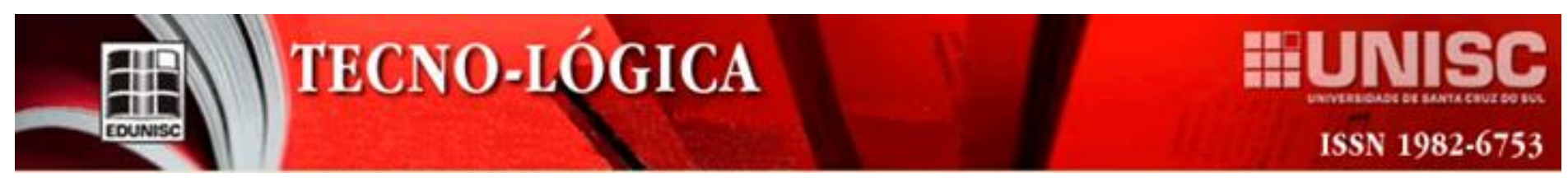

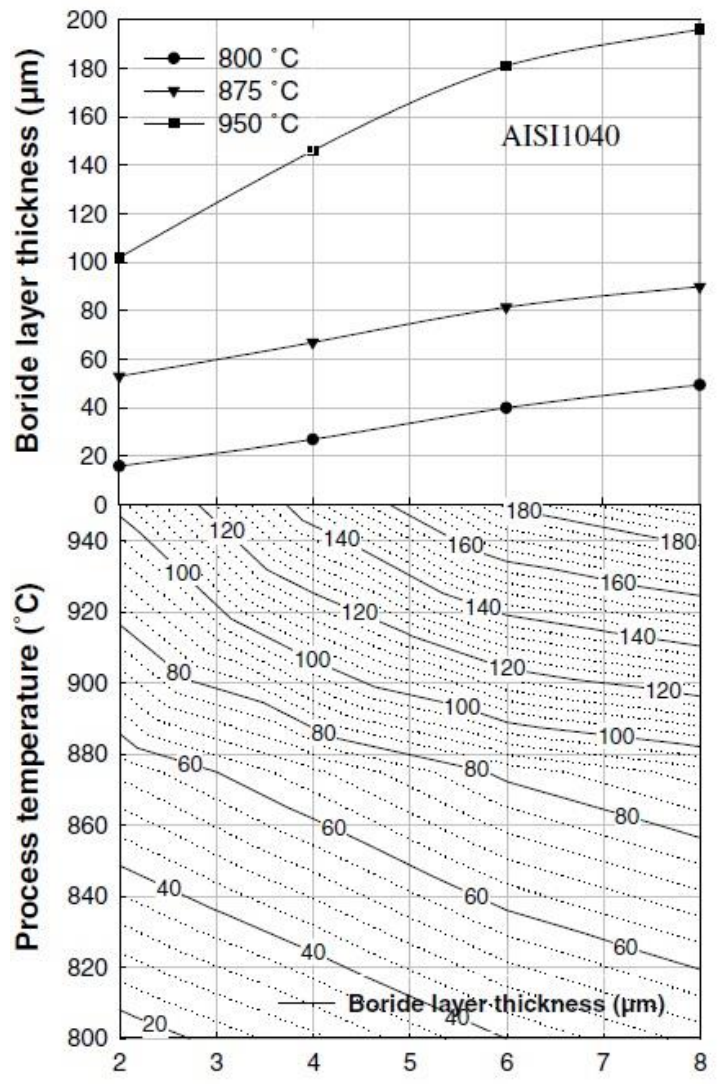

(a)

Treatment time (h)

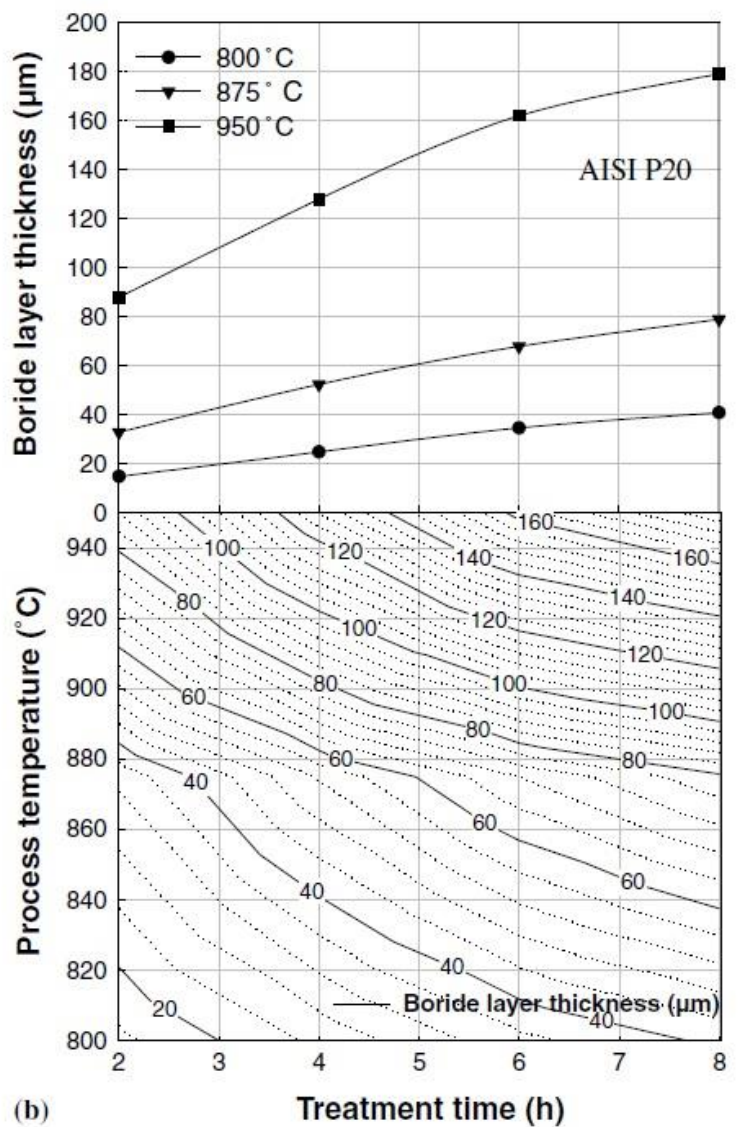

Figura 03 - Variação da profundidade da camada de Boreto para (a) Aço AISI 1040 e (b) Aço AISI P20 em função do tempo de processo, incluindo diagramas de contorno [13].

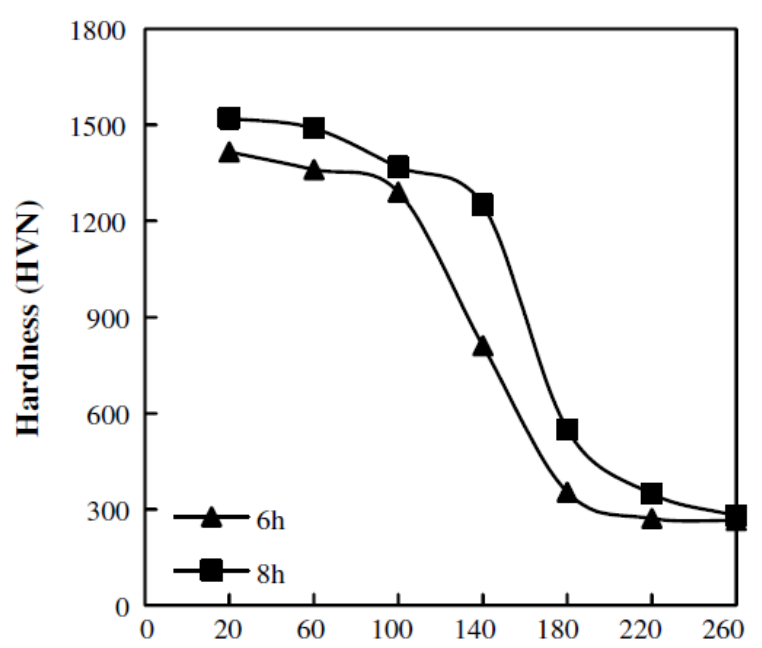

(a)
Distance from surface $(\mu \mathrm{m})$

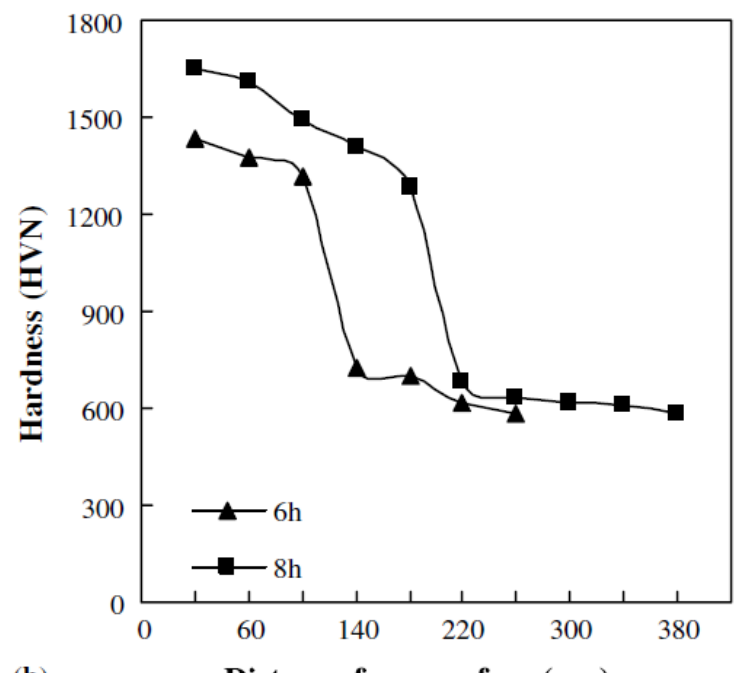

(b)

Distance from surface $(\mu \mathrm{m})$

Figura 04 - Variação da dureza dos boretos formados na superfície dos aços AISI 1040 (a) e AISI P20 (b). Boretados a 950 º, considerando o sentido da superfície para o interior [13]. 
Tabela 01 - Identificação de resultados, encontrados por diferentes pesquisadores, no que tange o Tratamento Termoquímico de Boretação.

\begin{tabular}{|c|c|c|c|c|c|c|c|}
\hline Ref. & Material & Agente Boretante & $\begin{array}{l}\text { Tempo } \\
\text { (h) }\end{array}$ & $\begin{array}{l}\text { Temp. } \\
\left({ }^{\circ} \mathrm{C}\right)\end{array}$ & $\begin{array}{l}\text { Dureza } \\
\text { Superf. }\end{array}$ & $\begin{array}{l}\text { Profund. } \\
\text { Camada } \\
(\mu \mathrm{m})\end{array}$ & $\begin{array}{l}\text { Boretos } \\
\text { Formados }\end{array}$ \\
\hline$[2]$ & ARMCO IRON & $\begin{array}{c}20 \% \mathrm{~B}_{4} \mathrm{C} ; 10 \% \mathrm{KBF}_{4} \\
70 \% \mathrm{SiC}\end{array}$ & 15 & 850 & $1490 \mathrm{HV}$ & 200 & $\mathrm{FeB} ; \mathrm{Fe}_{2} \mathrm{~B}$ \\
\hline$[3]$ & AISI D2 & $\begin{array}{c}85 \% \mathrm{Na}_{2} \mathrm{~B}_{4} \mathrm{O}_{7} \text { (Boráx) } \\
10 \% \mathrm{Fe}-\mathrm{Ti} ; 5 \% \mathrm{Al}\end{array}$ & 4 & 1000 & $1744 \mathrm{HV}$ & 120 & $\begin{array}{l}\mathrm{FeB} ; \mathrm{Fe}_{2} \mathrm{~B} \\
\mathrm{Fe}_{3} \mathrm{~B} ; \mathrm{Cr}_{2} \mathrm{~B}_{3}\end{array}$ \\
\hline$[6]$ & AISI P20 & EKabor ${ }^{\circledR}$ & 8 & 950 & $1650 \mathrm{HV}$ & 180 & $\begin{array}{l}\mathrm{FeB} ; \mathrm{Fe}_{2} \mathrm{~B} \\
\mathrm{MnB} ; \mathrm{CrB}\end{array}$ \\
\hline [13] & AISI 1040 & $\mathrm{~B}_{4} \mathrm{C} ; \mathrm{KBF}_{4} ; \mathrm{SiC}$ & 8 & 950 & $1500 \mathrm{HV}$ & 300 & $\mathrm{FeB} ; \mathrm{Fe}_{2} \mathrm{~B}$ \\
\hline [14] & AISI 1050 & EKabor ${ }^{\circledR}$ & 5 & 900 & $2261 \mathrm{HV}$ & 150 & $\mathrm{Fe}_{2} \mathrm{~B}$ \\
\hline [15] & $\begin{array}{c}\text { AÇO } \\
\text { FERRAMENTA }\end{array}$ & EKabor ${ }^{\circledR}$ & 2 & 1000 & $1854 \mathrm{HV}$ & 140 & $\mathrm{FeB} ; \mathrm{Fe}_{2} \mathrm{~B}$ \\
\hline [16] & AISI 1020 & $\begin{array}{c}5 \% \mathrm{~B}_{4} \mathrm{C} ; 5 \% \mathrm{KBF}_{4} \\
90 \% \mathrm{SiC}\end{array}$ & 4 & 1000 & $2100 \mathrm{HK}$ & 177 & $\mathrm{Fe}_{2} \mathrm{~B}$ \\
\hline [17] & AISI 4140 & $\begin{array}{c}65 \% \mathrm{Na}_{2} \mathrm{~B}_{4} \mathrm{O}_{7} \text { (Boráx) } \\
15 \% \mathrm{H}_{3} \mathrm{BO}_{3} ; 20 \% \mathrm{FeSi}\end{array}$ & 6 & 850 & $1446 \mathrm{HV}$ & 50 & $\begin{array}{l}\mathrm{FeB} ; \mathrm{Fe}_{2} \mathrm{~B} \\
\mathrm{CrB}\end{array}$ \\
\hline [18] & Aço XC38 & $\begin{array}{c}70 \% \mathrm{Na}_{2} \mathrm{~B}_{4} \mathrm{O}_{7}(\text { Boráx }) \\
30 \% \mathrm{~B}_{4} \mathrm{C}\end{array}$ & 4 & 950 & $2130 \mathrm{HV}$ & 150 & $\mathrm{FeB} ; \mathrm{Fe}_{2} \mathrm{~B}$ \\
\hline
\end{tabular}

\section{Conclusões}

Através da revisão bibliográfica que foi proposta, ao longo do presente trabalho, foi possível compreender os mecanismos e resultados obtidos através de um tratamento termoquímico de boretação. Trata-se de uma oportunidade muito relevante, no que diz respeito ao melhoramento de propriedades superficiais de aços largamente utilizados pela indústria ao longo das últimas décadas, sendo assim, justifica-se a importância de estudar profundamente as variáveis deste processo. A boretação pode ser extremamente explorada no que diz respeito ao agente boretante utilizado na realização do tratamento, sendo assim, acreditamos que explorar as mais diversas possibilidades de componentes para tal fim, possa proporcionar um avanço significativo no conhecimento que atualmente possuímos acerca desse assunto, o que poderá nos levar a conhecer e definir as condições ótimas para a realização deste tratamento, proporcionando a maior dureza superficial possível, o que certamente acarretará um melhor condicionamento ao desgaste do aço utilizado na fabricação de componentes submetidos a tal fenômeno.

\section{ANALYSIS OF THE EFFECTS OF THERMO- CHEMICAL TREATMENT BORETATION, APPLIED TO COMMERCIAL STEELS HAS BROAD INDUSTRIAL USE: REVIEW}

ABSTRACT: The improvement of studies, which allow the full understanding of the variables that involve the thermo-chemical treatment of boretation, has fundamental importance in order to progress in knowledge, leading to the implantation of such technique for industrial scale levels. Although it is a rich area within the context of scientific production, there are still many gaps to be uncovered with regard to boretation, especially as regards biting agents that can be used in the treatment, in addition to adequate temperature and time. The objective is to list the largest possible number of available data about the proposed theme, based on an updated bibliographical review, which will show that boretation, in fact, presents itself as an effective solution to avoid problems in components since it can lift up to five times the surface hardness of the steel base, which is used to manufacture them.

Keywords: boretation; literature review; steel; toughness.

\section{Referências}

[1] Davis J, Surface Hardening of Steels: Understanding the Basics. ASM International, Materials Park, OH, 2002.

[2] Martini C, Palombarini G, Poli G, Prandstraller D. Sliding and abrasive wear behaviour of boride coatings. Wear 2004(256):608-613.

[3] Oliveira C, Casteletti L, Totten G, Heck S. Production and characterization of boride layers on AISI D2 tool steel. Vacuum 2010(84):792-796. 
[4] Erdemir A, Bindal C. Formation and self-lubricating mechanisms of boric acid on borided steel surfaces. Surface and Coatings Technology 1995(7677):443-449.

[5] Genel K, Ozbek I, Kurt A, Bindal C. Boriding response of AISI W1 steel and use of artificial neural network for prediction of borided layer properties. Surface and Coatings Technology 2002(160):38-43.

6] Uslu I, Comert H, Ipek M, , Bindal C. Evaluation of borides formed on AISI P20 steel. Materials and Desing 2007 (28):55-61.

[7] Ucisik AH, Zeytin S, Bindal C. Boride coating on iron based alloys. Journal of the Australian Ceramic Society 2001 (37):83-94.

[8] Yoon J, Lee S, Jee Y. Plasma paste boronizing treatment of the stainless steel AISI 304. Surface and Coatings Technology 1999(1):71-75.

[9] Yang H, Wu X, Yang Z, Wang H. Enhanced boronizing kinetics of alloy steel assisted by surface mechanical attrition treatment. Journal of Alloys and Compounds 2014(590):388-395.

[10]. Balusamy T, Narayanan T, Park I, Lee M. Effect of surface mechanical attrition treatment (SMAT) on pack boronizing of AISI 304 stainless steel. Surface and Coatings Technology 2013(232):60-67.

[11] Xie F, Cheng J, Wang S. Effects and mechanisms of an alternating current field on pack boriding. Vacuum 2018 (148):41-47.

[12] Campos-Silva I, Flores-Jiménez M, Rodríguez-Castro G, Tadeo-Rosas R. Improved fracture toughness of boride layer developed with a diffusion annealing process. Surface and Coatings Technology 2013(237):429-439.

[13] Uslu I, Comert H, Bindal C. A comparison of borides formed on AISI 1040 and AISI P20 steels. Materials and Desing 2007(28):1819-1826.

[14] Meriç C, Sahin S, Yilmaz S. Investigation of the effect on boride layer of powder particle size used in boronizing with solid boron-yielding substances. Materials Research Bulletin, 2000(35):2165-2172.

[15] Sen S, Ozbek I, Sen U, Bindal C. Mechanical behaviour of borides formed on borided cold work tool steel. Surface and Coatings Technology 2001 (135):173-177.

[16] Krelling P, Costa C, Milan J, Almeida E. Micro-abrasive wear mechanisms of borided AISI 1020 steel. Tribology International 2017(111):234-242.

[17] Sen S, Sen U, Bindal C. The growth kinetics of borides formed on boronized AISI 4140 steel. Vacuum 2005(77):195-202.

[18] Allaoui O, Bouaouadja N, Sainderman G. Characterization of boronized layers on a XC38 steel. Surface and Coatings Technology 2006(201):3475-3482. 